\title{
Experience of Management of Anorexia Nervosa Patients With Extremely Severe Malnutrition In A Transdisciplinary Eating Disorders Inpatient Unit
}

Marie Guinhut ( $\square$ marieguinhut@live.fr)

Universite Paris Descartes https://orcid.org/0000-0002-4502-8416

Jean-Claude Melchior

Hopital Raymond-Poincare Unite de Nutrition

Nathalie Godart

Fondation Sante des Etudiants de France

Mouna Hanachi

Hopital Raymond-Poincare Unite de Nutrition

Research article

Keywords: anorexia nervosa, severe malnutrition, referral center, transdisciplinarity

Posted Date: May 1st, 2020

DOI: https://doi.org/10.21203/rs.3.rs-21423/v1

License: (c) (i) This work is licensed under a Creative Commons Attribution 4.0 International License.

Read Full License 


\section{Abstract}

\section{Background}

We describe a transdisciplinary medical unit, referral center for the management of anorexia nervosa (AN) adult patients with extremely severe malnutrition.

Method

History, organization and activity of the unit are described. Characteristics of all adult AN patients admitted for the first time in the unit between November 1997 and January 2014 and medical care provided are detailed, before discussing the value of such a unit.

Results

The unit was established as an independent department in 2004 and has 180 to 200 admissions per year. The patients hospitalized in the department are clinically serious and unstable because of life-threatening somatic complications due to a low BMI $\left(<13 \mathrm{~kg} / \mathrm{m}^{2}\right)$. They are referred on criteria of somatic severity. They often had multiple hospitalizations in other acute care hospitals before being supported in the unit. Among a cohort of 386 adult AN patients admitted for the first time in the unit, mean age was 29.4 ( \pm $11.5)$ years, mean BMI was $12.7( \pm 2.2) \mathrm{kg} / \mathrm{m}^{2}$ and $78.2 \%$ of patients had already been hospitalized in other hospitals.

Conclusion

This specialized transdisciplinary unit where physician nutritionists and psychiatrists coordinate medical care together, allow a better understanding and management of extreme malnutrition associated with AN. Thanks to their expertise, care teams are less critical and less rejecting towards patients. Thus, therapeutic alliance is optimized.

\section{Plain English Summary}

The question of where to hospitalize extremely malnourished anorexia nervosa (AN) patients is a real dilemma. On one hand, psychiatrists have to deal with severe medical complications that are not within their competences and that justify hospitalization in an internal medicine ward. On the other hand, medical doctors have to face psychic decompensations that would justify admission to a psychiatric ward.

In this context we would like to share our experience of management of severely malnourished AN adult patients in a transdisciplinary specialized eating disorders (ED) unit. Intensive medical care, both somatic and psychiatric, are provided thanks to a transdisciplinary therapeutic program, allowing a good therapeutic alliance between patient and caregiver, as indicated by a low dropout rate from inpatient treatment $(11.9 \%)$. 
The goals of treatment are to medically stabilize the patient, to initiate progressive refeeding and to start supportive psychotherapy before being transferred to a psychiatric ED unit.

This specialized transdisciplinary unit where physician nutritionists and psychiatrists coordinate medical care together, allow a better understanding and management of extreme malnutrition associated with AN. Thanks to their expertise, care teams are less critical and less rejecting towards patients. Thus, therapeutic alliance is optimized.

\section{Introduction}

With a Standardized Mortality Ratio (SMR) estimated at 5.86 (1), anorexia nervosa (AN) is a serious psychiatric disease whose mortality rate is the highest of all psychiatric pathologies (2). This mortality is especially high in tertiary care centers that treat the most severe forms of the disease (SMR at 10.6) (3). Indeed, AN can cause severe undernutrition, which can lead to medical complications and expose the patient to life-threatening risks. Thus, more than half of the deaths (54\%) of AN patients are attributable to the medical complications of AN, including undernutrition (4). Moreover, psychiatric co-morbidity often accompanies the undernutrition, which makes the patient's treatment more difficult to manage. Therefore, AN requires specific care. Most treatment programs provide medical and psychiatric components in the same milieu to optimize treatment to the extent possible.

But when a patient's nutritional status is too altered, (extremely severe undernutrition with BMI 8 to $13 \mathrm{~kg} / \mathrm{m}^{2}$ ) careful weight restoration with refeeding must be conducted in an inpatient medical facility to limit the risk of refeeding syndrome (RS). Nutritional rehabilitation often corresponds to "nutritional resuscitation" in extreme forms of AN. In addition, medical complications associated with psychiatric comorbidities are often worse and difficult to manage in a non-medical unit (5). Consequently, these units often refuse to accept these patients, or they discharge them prematurely.

The clinical nutrition - eating disorders (ED) - unit in Raymond Poincaré (RP) University- Hospital (Garches, 92380, France) is specialized in the management of extremely severe undernutrition and its complications associated with ED. The unit was established as an independent department in 2004, after a 10-year experience in the management of severe malnutrition resulting from different illnesses (specifically patients with HIV infection). It has been progressively oriented towards the exclusive care of patients with ED with life-threatening medical complications. Thanks to its expertise, the unit is now considered a regional and national "referral center" by the administrative authorities for the treatment of AN patients whose clinical condition can be serious and complicated. The unit is a partner of both the regional ED professional network, "Réseau TCA Francilien", and the national ED professional network, the "French Federation Anorexia Bulimia" (FFAB), associating all ED professionals in a collaborative network.

Our unit is a 15-bed unit in a conventional inpatient hospital setting.

In order to prevent runaways and suicide attempts, (which is high risk in compulsory hospitalisations), the unit is equipped with secure equipment and controlled access. It is only possible to enter or leave the unit 
by using a specific code (password). In addition, videophones allow caregivers to open the unit's entrance briefly, as necessary. To further enforce the security, all windows are locked and lined with an antiburglary glass, measures designed to protect the patients.

Two weekly outpatient consultations with a physician nutritionist as well as day-activities are carried out in the department. The mission of outpatient hospitalizations is to provide comprehensive clinical assessments, (complete nutritional assessment and screening for complications related to undernutrition), as well as post-hospitalization medical follow-up. It is a transdisciplinary outpatient clinic; a nurse, a dietician, a physician nutritionist, a psychiatrist and/or a psychologist, evaluates patients successively (or jointly).

The medical team is composed of two physicians, an assistant physician, two psychiatrists and two residents. The paramedical team consists of a pool of 10 nurses, 8 nursing assistants, a nursing supervisor, a clinical psychologist, a social worker, a dietician and a physiotherapist.

Our clinical nutritional rehabilitation team has developed expertise in treating extreme malnutrition due to severe AN. In order to deliver tailored care for the most severe cases of AN, we developed a transdisciplinary medical and psychiatric unit including both medical and psychiatric care in RP Hospital.

The goal of this paper is to present this transdisciplinary program of management of emaciated patients with severe forms of $\mathrm{AN}$ and the characteristics of patients hospitalized in the unit. We will explain the modalities of somatic and psychiatric care proposed, and we will describe patient characteristics and patient care pathways. We will then discuss the benefits of developing such a unit.

\section{li. Materials And Methods}

\section{II.1. Study Design and Aims}

We conducted a monocentric retrospective observational study in the cohort of adult AN patients admitted to the clinical nutrition-ED-unit in RP hospital for severe malnutrition and/or complications of the ED.

The primary objective was to specify characteristics of AN patients hospitalized in the unit: demographic, nutritional status, history of ED, care pathway. Secondary objective was to describe the modalities of somatic and psychiatric care proposed to AN patients hospitalized in the unit.

\section{II.2. Patients}

\section{II.2.1. Inclusion criteria}

We selected all patients hospitalized for the first time in the clinical nutrition-ED-unit in RP Hospital between November 1997 and January 2014, aged 15 years or older, diagnosed with AN according to the 
DSM IV criteria. The patient selection was provided by the hospital's department of statistics and medical information.

\section{II.2.2. Exclusion criteria}

We excluded from the study any patients who did not allow the use of their data for the study.

\section{II.2.4. Parameters studied}

For each patient, we performed a retrospective chart review and we recorded demographic and anamnesic data. Type of AN (restrictive, "AN-R" or purging/binging, "AN-BP”), duration of disease, purging behaviours, history of hospitalization for AN were detailed. Anthropometric data on admission and on discharge were noted as were patient psychiatric comorbidities, referral, length of stay and any intercurrent events which occurred during hospital stay.

\section{II.3. Procedures and ethical approval}

This study was conducted in accordance with the relevant French guidelines and regulations. It is part of a mortality study for which protocol was approved by the French data protection authority (CNIL, Commission Nationale de l'Informatique et des Libertés) and by two independent review boards (CCTIRS, Comité Consultatif sur le Traitement de I'Information en matière de Recherche dans le domaine de la Santé and CPP, Comité de Protection des Personnes). An information letter was sent to all patients selected for the study. Patient non-opposition was a prerequisite for the use of their data. Written informed consent for publication was obtained.

\section{II.4. Statistical analysis}

Analyses were performed with R software (version 3.5.3. 2019-03-11, R Foundation for Statistical Computing Platform: x86_64-w64-mingw32/x64 (64-bit)). Univariate statistics were used to describe the sample. Data were expressed as frequencies and percentages for nominal variables, and as means \pm standard deviations (SDs) for continuous variables.

\section{lii. Results}

\section{III.1. Patient characteristics}

Initially, 395 patients were selected but 9 of these were excluded because they declined use of their data in the study. Finally, we included a total number of 386 patients: 365 (94.6\%) were female and 21 (5.4\%) were male. Mean age at admission was 29.4 ( \pm 11.5 ) years old and $46.6 \%$ of patients had AN-R subtype while $48.2 \%$ had AN-BP subtype. Duration of AN at admission was $9.9( \pm 9.3)$ years. Before this first admission in the clinical nutrition-ED-unit, 302 (78.2\%) patients had already been admitted to other hospitals for AN. BMI at admission was $12.7( \pm 2.2) \mathrm{kg} / \mathrm{m}^{2}$. BMl at discharge was $14.2( \pm 1.9) \mathrm{kg} / \mathrm{m}^{2}$. During hospitalization, average weight gain was $3.8 \pm 4 \mathrm{~kg}(0.777 \mathrm{~kg}$ per week). Patient characteristics are presented in Table 1. 
Table 1. Patient characteristics $(\mathrm{N}=386)$.

\begin{tabular}{|c|c|}
\hline Characteristics & Mean \pm SD or $\quad$ N (Percentage) \\
\hline Female /Male & $365(94.6 \%) / 21(5.4 \%)$ \\
\hline Age at admission (years) & $29.4( \pm 11.5)$ \\
\hline \multicolumn{2}{|l|}{ Subtype of anorexia nervosa: } \\
\hline Restrictive & $180(46.6 \%)$ \\
\hline Binge purging & $186(48.2 \%)$ \\
\hline Atypical AN & $20(5.2 \%)$ \\
\hline Age at AN onset (years) & $19( \pm 7.6)$ \\
\hline Duration of AN at admission (years) & $9.9( \pm 9.3)$ \\
\hline History of hospitalization for AN in other hospitals (before admission in the unit) & $302(78.2 \%)$ \\
\hline Number of hospitalizations for AN (before admission in the unit) & $2.9( \pm 3.4)$ \\
\hline \multicolumn{2}{|l|}{ Patient's regular behavior: } \\
\hline Self induced vomiting & $162(42 \%)$ \\
\hline Laxative misuse & $81(21 \%)$ \\
\hline Potomania & $54(14 \%)$ \\
\hline Diuretic use & $15(3.9 \%)$ \\
\hline Problematic exercice & $175(45.3 \%)$ \\
\hline \multicolumn{2}{|l|}{ BMI (kg/mI): } \\
\hline Admission & $12.7( \pm 2.2)$ \\
\hline Discharge & $14.2( \pm 1.9)$ \\
\hline Weight gain during hospitalization (kg) & $3.8( \pm 4)$ \\
\hline \multicolumn{2}{|l|}{ Psychiatric comorbidities: } \\
\hline Personality disorders & $84(21.8 \%)$ \\
\hline Obsessive-compulsive disorders & $32(8.3 \%)$ \\
\hline Mood disorders and/or Anxiety disorders & $181(46.9 \%)$ \\
\hline History of suicide attempt & $81(21 \%)$ \\
\hline Self-mutilations (nonsuicidal self-injury disorder) & $28(7.3 \%)$ \\
\hline Attention Deficit Hyperactivity Disorder & $5(1.3 \%)$ \\
\hline Kleptomania & $3(0.8 \%)$ \\
\hline \multicolumn{2}{|l|}{ Addictions: } \\
\hline Alcohol use disorder & $34(8.8 \%)$ \\
\hline Substance use disorder & $24(6.2 \%)$ \\
\hline Tobacco use disorder & $127(33 \%)$ \\
\hline
\end{tabular}

\section{III.2. Patient's referral to the unit}

Among the patient cohort, the most frequent reason for admission was extremely severe undernutrition. Patients were sometimes hospitalized for other reasons such as the treatment of one or more medical complications related to undernutrition or to the process of refeeding itself initiated outpatient or inpatient in other hospitals. Short admissions were also organized for nutritional evaluation and development of a treatment regime. Finally, a few admissions were done in order to wean purging 
behaviours (self-induced vomiting, laxative or diuretic abuse). Distributions of reasons for hospitalization in the unit are presented in Table 2.

Table 2. Distribution of reasons for hospitalization in the unit $(\mathrm{N}=386)$.

\begin{tabular}{|l|l|}
\hline \multicolumn{1}{|c|}{ Reason for hospitalization } & N (Percentage) \\
\hline Severe undernutrition & $295(76.4 \%)$ \\
\hline Medical complications & $54(14 \%)$ \\
\hline Nutritional evaluation & $19(4.9 \%)$ \\
\hline Weaning of purging behaviours & $18(4.7 \%)$ \\
\hline
\end{tabular}

The protocol for the treatment of patients in the unit was individualised following an initial consultation or evaluation in psychiatric or general medicine day hospital. In case of emergency, the patient could be also admitted after a transfer from an inpatient unit in another hospital (e.g., an emergency room, medical ward, medical intensive care unit (MICU), or psychiatric ward), (refer to Table 3).

Table 3. Distribution of medical units that referred the patients to the unit $(n=366)$.

\begin{tabular}{|l|l|}
\hline Medical unit that referred the patients to the unit & N (Percentage) \\
\hline Psychiatric inpatient unit & $54(14.8 \%)$ \\
\hline Emergency department & $25(6.8 \%)$ \\
\hline Medical Intensive Care Unit & $65(17.8 \%)$ \\
\hline Medicine outpatient clinic & $100(27.3 \%)$ \\
\hline Psychiatric outpatient clinic & $59(16.1 \%)$ \\
\hline Medical ward & $51(13.9 \%)$ \\
\hline Student medical and psychiatric clinic & $2(0.5 \%)$ \\
\hline Adolescent care inpatient unit & $3(0.8 \%)$ \\
\hline Surgical ward & $1(0.3 \%)$ \\
\hline Obstetrics and gynecology inpatient unit & $2(0.5 \%)$ \\
\hline General pediatrics inpatient unit & $4(1.1 \%)$ \\
\hline
\end{tabular}

\section{III.3. Length of stay, Number of admission}

Our unit has 180 to 200 admissions per year with an average length of stay of $35.2( \pm 30.2)$ days. Patients can be hospitalized multiple times in the unit if necessary. Among the cohort, 127 (32.9\%) patients were re-hospitalized one or more times in the unit after their first admission because of a relapse of the disease resulting in a life-threatening clinical condition. 


\section{III.4. Inpatient Treatment and Management}

\section{III.4.1. Objectives of treatment in a full-time hospitalization}

Inpatient treatment has the following objectives:

- Begin a careful and gradual refeeding to stabilize critically ill patients suffering from severe malnutrition before they are transferred to a psychiatric inpatient unit specialized in ED. Psychiatric units are not usually sufficiently equipped and do not have the medical competence to handle the first stage of intensive care in case of severe malnutrition (6).

- Diagnose and treat any medical complications.

- Initiate, continue or resume psychiatric care. The objectives of psychiatric intervention at this stage of undernutrition are limited to: detecting and preventing any suicidal behaviour, taking security measures to protect the patient if necessary, providing supportive therapy and creating specific a short-term and long-term treatment plan.

- Decrease invasive binging and purging behaviors, (self-induced vomiting, laxative or diuretic abuse), potomania, and problematic exercising, as necessary.

- Effort to preserve and improve the socio-professional integration of the patient (if deficient) in anticipation of his discharge from the hospital.

To achieve these goals, medical care should be multifocal and therefore transdisciplinary including meetings to coordinate planning. Two different transdisciplinary staff meetings are organized regularly to optimize and coordinate patient care:

- Weekly staff meeting attended by nurses, auxiliary nurses, medical doctors, psychiatrists, residents, psychologist, physiotherapist, and all students

- Monthly staff meeting coordinated by a psychologist and a psychiatrist to discuss the difficulties encountered by the medical and paramedical team in providing patient care; psychological and psychiatric support help the team deal with difficulties: improve coping, discuss the emotional impact of working with this patient population, strengthen the collective dimension of the team.

In addition, the therapeutic alliance between the patient and the healthcare team is essential to the efficacy of the treatment program. Ideally, (except in cases of emergency hospitalization), the conditions of hospitalization are discussed in advance with the patient and his family during a pre-admission consultation.

\section{III.4.2. The individualized therapeutic program}

A written care contract is agreed between the patient and his caregivers and signed by both. It sets out weighted objectives and the steps needed to combat anorexic cognitions. The conditions of 
hospitalization are also detailed. They are individualised according to the patient, depending on the severity of his symptoms and the psychiatric comorbidities. The limited access to sanitary facilities is outlined and explained in the contract including the prevention of postprandial vomiting compulsions and food spills, as well as naso-gastric tube and feeding by EN. Access to mobile phones, use of laptops, access to the Internet, frequency of family visits and off-premises passes are also determined in agreement with the patient, with the patient's family (as appropriate) and detailed in the contract.

Discharge planning for the continuation of medical care (specifying the medical team and treatment facility) is determined progressively based on discussions with the patient and his family as clinical improvement of the patient progresses.

\section{III.4.3. Enteral nutrition}

When a patient's BMI is below 13 , artificial nutrition support is usually indicated to initiate the refeeding of the patient (7). However, in the unit, when a patient's BMI is between 12 and 13, the prescription of artificial nutrition is discussed and decided according to the patient's situation, taking into consideration his clinical condition (presence of edema, physical exhaustion), his calorie intake and his blood test results (8). The existence of medical complications weighs in favor of instituting nutritional support therapy. When a patient's BMI is less than 12, artificial nutrition is proposed from the start (8).

Assuming the patient has an accessible and intact digestive tract (which is true in most cases) (9), the choice of nutritional support is typically enteral nutrition (EN) administered via a small-caliber nasogastric tube as recommended $(6,10)$. Parenteral nutrition is not used nor recommended in AN $(11,12)$, except in cases where intestinal pathology makes EN impossible. PN exposes the patient to a risk of infectious, mechanical and metabolic complications (13). Vomiting is not a contraindication to EN.

The good tolerance of EN (14), its effectiveness on both weight gain (a weight gain between $500 \mathrm{~g}$ and 1 $\mathrm{kg}$ per week is recommended (6)) and on the reduction of the duration of hospitalizations, have been demonstrated (15) in AN malnourished patients. EN is therefore widely prescribed in the unit. In our cohort, 316 (81.9\%) patients benefited from EN during their hospital stay.

EN is prescribed in the following manner:

- A standard polymeric product, isocaloric (providing $1 \mathrm{kcal} / \mathrm{ml}$ ), normoprotidic, without fiber is most often prescribed

- In cases of impaired renal function or particularly in very low weight patients, a paediatric product less rich in protein is preferred.

To improve its performance and clinical and metabolic tolerance, EN is administered in a continuous flow over 24 hours using a flow control pump. When undernutrition is severe $(\mathrm{BMI}<12)$, refeeding is exclusively enteral in the first days. An oral diet is gradually introduced later (see dietary management). 
Calorie intake is started at $10 \mathrm{kcal} / \mathrm{kg}$ body weight / 24 hours during the first 48 hours as recommended with severely malnourished patients (16). Then it is increased gradually, in increments of $250 \mathrm{kcal}$, according to the patient's clinical and biological tolerance, to reach $45 \mathrm{kcal} / \mathrm{kg}$ body weight/ 24 hours.

\section{III.4.4. Hydration, vitamins and trace elements}

During the first 48 hours of hospitalization, intravenous supplementation with vitamins, trace elements and phosphorus is carried out to correct potential micronutrient deficiencies and to prevent RS as recommended (16). Intravenous rehydration with a $5 \%$ glucose polyionic solution is also administered, limited to $30 \mathrm{ml} / \mathrm{kg}$ body weight / 24h. Patients are put on a low sodium diet (sodium intake $<1 \mathrm{mmol} /$ $\mathrm{kg}$ body weight / $24 \mathrm{~h}$ ) to prevent water inflation. Daily intake of phosphorus, vitamins and trace elements is continued orally.

Additional intravenous contributions of potassium, magnesium and phosphorus are added in case of hypokalemia, hypomagnesemia or hypophosphatemia.

\section{III.4.5. Oral nutrition}

Dietary management is an integral part of overall care and it becomes more and more important with the progression of the patient's hospitalization, in parallel with the decrease in EN. The reintroduction of oral feeding is done gradually, once refeeding has begun and in the absence of critical metabolic abnormalities, at a rate adapted to the physiological and psychological capacities of the patient. The reintroduction of food must be progressive, as under-nutrition is severe.

Protein foods are re-introduced last (after vegetables and carbohydrates), in order to limit protein intake at a level under $2.5 \mathrm{~g} / \mathrm{kg}$ of body weight per day. Oral feeding is carried out with 3 meals per day and, if necessary, a snack at $4 \mathrm{pm}$. The meal duration must not exceed 30 minutes for lunch and 45 minutes for dinner.

A food-monitoring sheet is displayed in the patient's room which notes the current oral intakes of the patient. Once established, the composition of the meal can only be changed after the dietician has interviewed the patient. As oral nutrition and weight gain are acquired, enteral caloric intakes are progressively reduced. However, we have observed that more than one-third of patients are still on EN on discharge. Among our cohort, 137 (35.5\%) patients were still receiving EN when they left the unit. Hence, EN was ongoing in their transfer unit to continue the refeeding process. It is recommended to interrupt EN if the oral intake is satisfactory when the BMI is around $14 \mathrm{~kg} / \mathrm{m} \mathrm{(16).}$

\section{III.4.6. Prevention of refeeding syndrome}


The severe and chronic undernutrition of the AN patient $(\mathrm{BMI}<16)$ exposes the patient to an increased risk of RS at the initial phase of refeeding $(6,17)$. The metabolism of the patient, which was idling in a state of adaptation to prolonged fasting, faces a new situation: the reintroduction of nutrients and its resulting increase in insulin levels (18). This metabolic change puts the patient at risk, especially if the increased calorie intake is not introduced gradually with movement of water and electrolytes from the extracellular sector to the intracellular sector. RS can manifest with ionic disorders, (mainly hypophosphatemia, hypokalemia and/or hypomagnesemia), fluid retention and one or more organ dysfunction(s) (acute heart failure, renal failure, respiratory failure, liver failure, convulsion or even coma) (19).

To prevent RS, various measures are implemented:

- Refeeding is started with a minimum calorie intake, then increased very gradually, following the rule "start slow, advance slow." (18)

- Adjustment of calorie intake is individualized to the patient's metabolic tolerance and weight gain. Ideally, the weight gain should be 0.5 to $1 \mathrm{~kg} /$ week $(6,18)$

- Supplementation with multivitamins, trace elements, potassium, phosphorus, and magnesium is started empirically from the first day of admission, and then adapted, based on the biological results.

- Comprehensive clinical monitoring with special attention to heart rate, edema and hydration status.

- Regular biological monitoring (initially daily) of blood glucose, creatinine, liver enzymes, plasma electrolytes, and phosphorus $(6,18)$.

\section{III.4.7. Psychotropic treatment}

There is no specific psychotropic treatment for $\operatorname{AN}(6,20)$ and those medications can have severe side effects in case of malnutrition. This is the reason why psychotropic medications are not systematically ordered in the unit. However, as recommended in all international guidelines (20), antidepressant medications are sometimes used when the patient still presents anxious depressive symptoms leading to an anxious or depressive disorder diagnosis despite improvement in his nutritional status (22). When the patient's anxiety symptoms are so severe that it is a barrier to his refeeding process, a small dose of anxiolytics can also be prescribed.

\section{III.4.8.Prevention of decubitus ulcers and deep vein thrombosis}

Strict bed rest is ordered in the beginning of hospitalization. It can be extended for some patients. Daily nursing care provided by nurses and nursing aids, and the use of air mattresses are the main measures to prevent bedsores in the unit. In addition, a prophylactic dose of anticoagulant treatment is ordered to prevent deep vein thrombosis. 


\section{III.4.9. Blood analysis and imaging}

Some analyses are carried out during the early phase of the hospital stay:

- Blood analysis (CBC, platelets count, electrolytes, urea, creatinine, calcium, phosphorus, magnesium, albumin, transthyretin, glycemia, liver enzymes, PT, PTT, CRP, Thyroid Function Tests, folate, vitamin B12, vitamins A, D and E, zinc, copper, selenium)

- ECG

- Chest X Ray to verify the NGT position; (if an NGT is inserted)

A bone densitometry is done to assess the patient's bone mineralization status and to detect potential osteoporosis. It may be the first time a patient has had a bone densitometry in his medical history. If the patient had already a bone densitometry done in the past, a waiting period of two years is needed before repeating it (6).

An echocardiogram is also provided in the presence of clinical signs of cardiac insufficiency and/or ECG abnormality.

\section{III.4.10. Clinical and bio-clinical monitoring}

An internal medicine doctor and a psychiatrist perform daily ward rounds together. All the patients present in the ward are seen every day by the team of doctors who provide physical examinations, discussions about hospitalization conditions, adjustment and revaluation of treatment, management of acute medical issues. Thus, each medical complication is managed adequately.

Complementary investigations are repeated according to patient needs and changes, but systematically, three to six times per day, capillary blood glucose monitoring and vital signs monitoring are provided. Indeed, an isolated tachycardia can be an early sign of sepsis or cardiac dysfunction. Other complications include patients who are hypothermic and others who develop severe hypoglycemia because of severe malnutrition.

Body weight monitoring is done twice a week, on fixed days, before breakfast with patients wearing only their underwear.

During the first week of hospitalization, daily blood tests are performed specifically for phosphorus, electrolytes, creatinine and liver enzymes monitoring. Later, blood tests are repeated once a week if there is no acute abnormality.

A Nitrogen balance is done regularly to check the patient metabolic status, which should be anabolic during the refeeding process. 


\section{III.4.11. Psychiatrist's role}

Psychiatrists assess the psychiatric comorbidities. They manage acute psychiatric symptoms. They are responsible for prescription of psychotropic medications when needed, according to the patient's biological and clinical tolerance. In cases of compulsory hospitalization, they write medical certificates in collaboration with the medial doctor.

In cooperation with psychologists, psychiatrists provide supportive psychotherapy. Structured therapy is difficult and may be impossible in case of extremely severe malnutrition. In fact, some psychiatric symptoms can be caused by malnutrition. Moreover, in the early phase of hospital, cognitive functions are altered by undernutrition itself (18).

\section{III.4.12. Psychologist's role}

The psychologist is responsible for individual psychotherapy support for each patient at least once a week. The frequency of these interviews is adjusted taking into account both the patient's preferences and the medical and psychiatric teams' evaluation. The psychologist also coordinates group activities or occupational therapy ( 3 times per week) to enable the patient to build alliances with the team and develop social relationships based on mediational activities. Different themes are explored through these activities. The different workshops include: group therapy focused on life and treatment in the unit, a newspaper workshop, cultural mediation, creative arts, and writing. Patients are invited to participate as soon as their physical conditions allow it.

\section{III.4.13. Medical management difficulties}

Because of the extreme severity of the patients' medical conditions and despite all the precautions taken during the refeeding process, some acute medical complications can appear. It was rarely a refeeding syndrome ( 23 cases in our cohort- $5.9 \%$ of patients); most often, these complications corresponded to: hemodynamic instability, electrolyte imbalance, anemia, severe neutropenia, acute organ dysfunction (cardiac, hepatic, renal) or sepsis linked to the relative immunosuppression induced by the malnutrition.

All these situations are managed individually. In cases of critical or unstable clinical parameters or vital organ dysfunction, a transfer to the MICU is organized.

Among our cohort, 99 (25.6\%) patients were transferred to the MICU during their hospitalization in the unit. Reasons for these MICU transfers are presented in Table 4.

Consultations to other sub-specialties such as infectious diseases or hepatology may also be required to optimize the management of patients at times. 


\begin{tabular}{|l|l|l|}
\hline \multicolumn{1}{|c|}{$\begin{array}{c}\text { Reason for medical intensive care unit (MICU) transfer during } \\
\text { hospitalization in the unit }\end{array}$} & $\begin{array}{c}\text { Number of } \\
\text { patients }\end{array}$ & \multicolumn{1}{c|}{$\begin{array}{c}\text { Percentage of the total number of MICU } \\
\text { transfers (N=99) }\end{array}$} \\
\hline Hypokalemia complicated by ECG changes & 21 & $21,2 \%$ \\
\hline Hypophosphatemia complicated by ECG changes & 11 & $11,1 \%$ \\
\hline Severe hyponatremia & 9 & $9,1 \%$ \\
\hline Severe hypertransaminasemia and/or hepatic failure & 20 & $20,2 \%$ \\
\hline Acute cardiac failure & 6 & $6,1 \%$ \\
\hline Hemodynamic instability and / or cardiac arrhythmias & 24 & $24,2 \%$ \\
\hline Hypothermia & 5 & $5 \%$ \\
\hline Acute renal failure & 10 & $10,1 \%$ \\
\hline Suicide attempt & 8 & $8,2 \%$ \\
\hline Acute pancreatitis & 1 & $1 \%$ \\
\hline Severe sepsis & 14 & $14,1 \%$ \\
\hline Anasarca & 3 & $3 \%$ \\
\hline Severe neurological disorders & 5 & $5 \%$ \\
\hline Severe symptomatic hypoglycemia & 6 & $6,1 \%$ \\
\hline Gastric distension & 1 & $1 \%$ \\
\hline Parenteral nutrition use & 1 & $1 \%$ \\
\hline
\end{tabular}

\section{III.4.14. Psychiatric care difficulties}

AN is primarily a psychiatric disorder characterized by poor compliance, opposition to care and denial of the severity of the clinical condition by the patient. These situations can lead to some runaways ( 9 cases in our cohort - $2.3 \%$ of patients) and to discharges against medical advice before the end of the care protocol (46 cases among our cohort $-11.9 \%$ of patients without immediate life-threatening conditions). However, in cases of vital risk, (when the patient's condition is compromised and the continuation of refeeding is urgently required), if the patient does not agree to the continuation of care, a legal procedure for compulsory treatment under French Law can be implemented in the unit. This procedure is developed in collaboration with the regional reference psychiatric center and implemented in our medical unit until the clinical state of the patient allows other modalities of treatment. In our cohort, 24 (6.2\%) patients were affected by this procedure.

Some factors make hospitalization difficult for the patient including following the treatment plan, conforming to hospital regulations. Weight gain can be anxiogenic and patients frequently develop problematic physical activity.

In addition, AN patients admitted in the unit had frequent psychiatric comorbidities (refer to Table 1). Anxiety, depression and personality disorders were the most frequent disorders observed and were much more frequent than in less severe samples $(22,23)$. 


\section{III.4.15. Discharge from the unit and continuation of care}

When a patient leaves the zone of critical danger, and his clinical condition is stable, he can be discharged. The refeeding process should achieve a minimum BMI of 13 . From then on, usually patients are transferred to an ED psychiatric unit to continue the refeeding process and initiate ED specialised therapies. This transfer is organized when the patient agrees. But some patients are unwilling to accept the transfer. When this occurs, there are two possibilities: if their clinical state is still critical, a compulsory treatment is implemented in the ED psychiatric unit; if not, they are discharged to ambulatory treatment. When the transfer takes place, it is preceded by a pre-admission consultation or 2 to 3-day hospital sessions in the designated psychiatric unit. Follow-up care can also be provided in a conventional psychiatric unit. In our cohort, $44 \%$ of patients benefited from a transfer to a psychiatric ward after their hospitalization in the unit (refer to table 5). In all cases, medical follow-up is organized in consultation and/or outpatient hospitalization in the clinical nutrition-ED- unit in RP hospital.

Table 5. Distribution of facilities receiving patients after discharge from the unit $(\mathrm{N}=386)$.

\begin{tabular}{|l|l|}
\hline Facility receiving the patient after discharge from the unit & N (Percentage) \\
\hline Home & $169(43.8 \%)$ \\
\hline General psychiatry & $45(11.7 \%)$ \\
\hline Psychiatric unit specialized in eating disorders & $116(30.1 \%)$ \\
\hline Medicine rehabilitation center & $6(1.5 \%)$ \\
\hline Nutrition rehabilitation center & $8(2.1 \%)$ \\
\hline Psychiatry Day Hospital & $14(3.6 \%)$ \\
\hline Endocrinology department & $1(0.3 \%)$ \\
\hline Surgical ward & $4(1 \%)$ \\
\hline Psychiatric rehabilitation center & $9(2.3 \%)$ \\
\hline Internal medicine ward & $3(0.8 \%)$ \\
\hline Clinical nutrition unit & $1(0.3 \%)$ \\
\hline Home hospitalization & $1(0.3 \%)$ \\
\hline Obstetrics and gynecology department & $2(0.5 \%)$ \\
\hline Medical Intensive Care Unit & $1(0.3 \%)$ \\
\hline Death during the hospital stay & $6(1.5 \%)$ \\
\hline & \\
\hline
\end{tabular}

\section{Iv. Discussion}

The question of where to hospitalize extremely malnourished AN patients is a real concern raised in the literature by psychiatric teams specialized in the management of AN (24) (25). It may seem like a dilemma. On one hand, in case of extreme malnutrition related to AN, psychiatrists have to deal with severe medical complications that are not within their competences and that justify hospitalization in an internal medicine ward. On the other hand, medical doctors have to face the resistance to treatment inherent to AN patients (denial, treatment refusal, ambivalence about treatment) » and psychic decompensations that would justify admission to a psychiatric ward (24). This problem is mentioned in the MARSIPAN report which specifies that specialist ED units (SEDUs) are not suitable for treating severe medical complications (25). Thus, a survey in SEDUs published by the MARSIPAN working group showed 
that services SEDU could not offer intravenous infusion, parenteral nutrition and treatment of serious medical complications. Patients with electrolyte or renal abnormalities or comorbidity increasing the risk of refeeding syndrome should not be managed in a SEDU. MARSIPAN guidelines recommend to transfer these patients to a medical ward. Then there are the issues of liaison and transfer between the two settings (SEDU and medical ward) (25).

Our unit responds to this problem by providing both somatic and psychiatric care, in the same place, to make the management of severely malnourished AN patients more safe and effective.

The development of a team specialized in the management of severe malnutrition and the regular admission of AN patients in the unit has allowed us to develop expertise in the most extreme clinical situations. As explained by E. S. Chu (26), the management of patients with complex illnesses, is better when it is provided by a medical team with specific expertise. In this context, transdisciplinary management involving psychiatrists and medical doctors appears essential. Indeed, the goal of having a transdisciplinary team is to respect the patient's individuality by proposing both a global and a personalized approach. The team-work of the different professionals who exchange ideas and mutually enrich each other makes it possible to offer optimal care for the patient.

The other specific benefits of transdisciplinarity are the possibility of: initiating early psychiatric followup, ensuring continuity of care by preparing for subsequent care in specialized ED psychiatric unit, initiating compulsory treatment if necessary. Thanks to the centralization of treatment for difficult medical situations, the unit has become an important referral center for effective treatment of serious ED.

Treatment results suggest that the therapeutic alliance between the patient and the healthcare team is better, as evidenced by the low drop-out rate estimated at $11.9 \%$ in a sample of 386 patients hospitalized in the unit, compared to $20.2-57.6 \%$ in the literature (27).

The development of the therapeutic alliance is favored by the involvement of the close entourage of patients in the care program. The involvement of the families is an important factor in patient adherence to care (28).

Optimization of the medical care of patients is also illustrated through the low rate of RS occurring during hospitalization, despite cases of severe malnutrition (23 cases of RS out of 386 patients, a rate of $5.9 \%$ ).

Systematic review of the literature did not identify, either in France or elsewhere in Europe, a similar unit providing medical and psychiatric transdisciplinary care to patients with severe forms of AN. However, we did find an acute medical unit in Denver accepting patients with ED and severe medical complications (26). This unit does not have an onsite psychiatrist but psychologists can support the patients during their hospitalization. In Munich, there is also a psychiatric intensive care unit accepting severely malnourished AN patients for refeeding since 2000 (29).

We share the opinion of E.S.Chu (26) that this rare, medically unstable and complex population of patients with severe malnutrition secondary to AN requires a specific medical management by a highly 
specialized multidisciplinary team within a referral center. The team also has a mission, through clinical research perspectives, to provide a better understanding of the somatic complications associated with extreme malnutrition and thus participate in the elaboration of protocols and management guidelines (6, 30).

\section{Conclusion}

The transdisciplinary model of management and treatment of adult patients suffering from AN in the clinical nutrition-ED-unit in Raymond Poincaré-University Hospital could, if it were available more widely, benefit the most distressed patients and potentially decrease complications and mortality related to severe AN and promote patient care compliance. Thanks to their expertise, specialized care teams are very competent, less critical and less rejecting towards patients (31).

\section{Abbreviations}

AN

anorexia nervosa

AN-R

anorexia nervosa restrictive subtype

AN-BP

anorexia nervosa binge purging subtype

$\mathrm{BMI}$

body mass index

ED

eating disorders

EN

enteral nutrition

MARSIPAN

management of really sick patients with anorexia nervosa

MICU

medical intensive care unit

RP

Raymond Poincaré

RS

refeeding syndrome

SEDU

specialist eating disorders unit

\section{Declarations}


Ethical approval and consent to participate: All procedures performed in studies involving human participants were in accordance with the ethical standards of the institutional and/or national research committee and with the 1964 Helsinki declaration and its later amendments or comparable ethical standards.

Informed consent was obtained from all individual participants included in the study.

Consent for publication: Written informed consent for publication was obtained (please refer to paragraph: II.3. Procedures and ethical approval).

Availability of data and materials: The datasets generated and analysed during the current study are not publicly available due to other works being under progress but are available from the corresponding author on reasonable request.

Competing interests: The authors declare that they have no competing interests.

Funding: Not applicable.

Authors' contributions: MG collected and analysed the patients' data and wrote the initial manuscript. JCM, NG and MH analysed the patients' data and contributed in writing the manuscript. All authors read and approved the final manuscript.

Acknowledgements: Not applicable.

\section{References}

1. Arcelus J, Mitchell AJ, Wales $\mathrm{J}$, Nielsen S. Mortality rates in patients with anorexia nervosa and other eating disorders. A meta-analysis of 36 studies. Arch Gen Psychiatry juill. 2011;68(7):724-31.

2. Chesney E, Goodwin GM, Fazel S. Risks of all-cause and suicide mortality in mental disorders: a meta-review. World Psychiatry Off J World Psychiatr Assoc WPA juin. 2014;13(2):153-60.

3. Huas C, Caille A, Godart N, Foulon C, Pham-Scottez A, Divac S, et al. Factors predictive of ten-year mortality in severe anorexia nervosa patients. Acta Psychiatr Scand janv. 2011;123(1):62-70.

4. Sullivan PF. Mortality in anorexia nervosa. Am J Psychiatry juill. 1995;152(7):1073-4.

5. Evans DL, Charney DS, Lewis L, Golden RN, Gorman JM, Krishnan KRR, et al. Mood disorders in the medically ill: scientific review and recommendations. Biol Psychiatry 1 août. 2005;58(3):175-89.

6. Haute Autorité de santé. Anorexie mentale: prise en charge. Recommandations de bonne pratique, juin 2010. Nutr Clin Métabolisme juin. 2011;25(2):57-74.

7. Paccagnella A, Mauri A, Baruffi C, Berto R, Zago R, Marcon ML, et al. Application criteria of enteral nutrition in patients with anorexia nervosa: correlation between clinical and psychological data in a " lifesaving » treatment. JPEN J Parenter Enteral Nutr juin. 2006;30(3):231-9. 
8. Hanachi-Guidoum M. [Treating the somatic complications of anorexia]. Soins Psychiatr déc. 2016;37(307):34-7.

9. Martínez-Olmos MA, Peinó R, Prieto-Tenreiro A, Lage M, Nieto L, Lord T, et al. Intestinal absorption and pancreatic function are preserved in anorexia nervosa patients in both a severely malnourished state and after recovery. Eur Eat Disord Rev J Eat Disord Assoc mai. 2013;21(3):247-51.

10. Practice guideline for the treatment of patients with eating disorders (revision). American Psychiatric Association Work Group on Eating Disorders. Am J Psychiatry janv. 2000;157(1 Suppl):1-39.

11. National Collaborating Centre for Mental Health, éditeur. Eating disorders: core interventions in the treatment and management of anorexia nervosa, bulimia nervosa and related eating disorders; National Clinical Practice Guideline No. CG9. Leicester: British Psychological Society [u.a.]; 2004. 260 p.

12. Garber AK, Sawyer SM, Golden NH, Guarda AS, Katzman DK, Kohn MR, et al. A systematic review of approaches to refeeding in patients with anorexia nervosa. Int J Eat Disord mars. 2016;49(3):293310.

13. Melchior J-C, Corcos M. Parenteral Nutrition and Anorexia Nervosa: Is It Useful, Is It Ethical? J Adolesc Health avr. 2009;44(4):410-1.

14. Gentile MG. Enteral nutrition for feeding severely underfed patients with anorexia nervosa. Nutrients. 2012;4(9):1293-303.

15. Rigaud D, Brondel L, Poupard AT, Talonneau I, Brun JM. A randomized trial on the efficacy of a 2month tube feeding regimen in anorexia nervosa: A 1-year follow-up study. Clin Nutr Edinb Scotl août. 2007;26(4):421-9.

16. Royal College of Psychiatrists London

Guidelines for the nutritional management of anorexia nervosa [Internet] Disponible sur:

Royal College of Psychiatrists London. Guidelines for the nutritional management of anorexia nervosa [Internet]. 2005 oct [cité 26 juin 2019] p. 396-396. Disponible sur: https://www.cambridge.org/core/product/identifier/S0955603600029408/type/journal_article.

17. Nutrition Support for Adults. Oral Nutrition Support, Enteral Tube Feeding and Parenteral Nutrition. National Collaborating Centre for Acute Care (UK); 2006.

18. Mehler PS, Winkelman AB, Andersen DM, Gaudiani JL. Nutritional Rehabilitation: Practical Guidelines for Refeeding the Anorectic Patient. J Nutr Metab. 2010;2010:1-7.

19. Rio A, Whelan K, Goff L, Reidlinger DP, Smeeton N. Occurrence of refeeding syndrome in adults started on artificial nutrition support: prospective cohort study. BMJ Open. 2013;3(1):e002173.

20. Aigner M, Treasure J, Kaye W, Kasper S, WFSBP Task Force On Eating Disorders. World Federation of Societies of Biological Psychiatry (WFSBP) guidelines for the pharmacological treatment of eating disorders. World J Biol Psychiatry Off J World Fed Soc Biol Psychiatry sept. 2011;12(6):400-43.

21. version française du SCOFF S-F. Anorexie mentale: prise en charge. Recommandations de bonne pratique, juin 2010. Nutr Clin Métabolisme. 2011;25:57-74. 
22. Leblé N, Radon L, Rabot M, Godart N. [Depressive symptoms during anorexia nervosa: State of the art and consequences for an appropriate use of antidepressants]. L'Encephale févr. 2017;43(1):62-8.

23. Godart NT, Perdereau F, Jeammet P, Flament MF. [Comorbidity between eating disorders and anxiety disorders: results]. L'Encephale avr. 2005;31(2):152-61.

24. Saito S, Kobayashi T, Kato S. Management and treatment of eating disorders with severe medical complications on a psychiatric ward: a study of 9 inpatients in Japan. Gen Hosp Psychiatry juin. 2014;36(3):291-5.

25. Robinson P, Rhys Jones W. MARSIPAN: management of really sick patients with anorexia nervosa. BJPsych Adv janv. 2018;24(1):20-32.

26. Chu ES, Gaudiani JL, Mascolo M, Statland B, Sabel A, Carroll K, et al. ACUTE center for eating disorders. J Hosp Med avr. 2012;7(4):340-4.

27. Wallier J, Vibert S, Berthoz S, Huas C, Hubert T, Godart N. Dropout from inpatient treatment for anorexia nervosa: critical review of the literature. Int J Eat Disord nov. 2009;42(7):636-47.

28. Rienecke RD, Richmond R, Lebow J. Therapeutic alliance, expressed emotion, and treatment outcome for anorexia nervosa in a family-based partial hospitalization program. Eat Behav. 2016;22:124-8.

29. Born C, de la Fontaine L, Winter B, Müller N, Schaub A, Früstück C, et al. First results of a refeeding program in a psychiatric intensive care unit for patients with extreme anorexia nervosa. BMC Psychiatry 24 mars. 2015;15:57.

30. Hanachi M, Melchior JC, Crenn P. Hypertransaminasemia in severely malnourished adult anorexia nervosa patients: risk factors and evolution under enteral nutrition. Clin Nutr Edinb Scotl juin. 2013;32(3):391-5.

31. Treasure J, Crane A, McKnight R, Buchanan E, Wolfe M. First do no harm: iatrogenic maintaining factors in anorexia nervosa. Eur Eat Disord Rev J Eat Disord Assoc août. 2011;19(4):296-302. 